\title{
LA CONSTRUCCIÓN SOCIAL DE SIGNIFICADOS EN EL FIN DE LA ERA DEL PADRE
}

\section{THE SOCIAL CONSTRUCTION OF MEANING AT THE END OF THE ERA OF FATHER}

\author{
María Flórez-Estrada*
}

\begin{abstract}
RESUMEN
El presente artículo tiene como propósito reflexionar sobre la construcción social de los significados a partir de la eclosión del mundo de la vida en la actualidad posmoderna. Además, propongo que esta eclosión hace posible un salto epistemológico que podría viabilizar el tránsito del "reino de la necesidad al reino de la libertad" en el plano de la cultura y de las subjetividades. Lo hago principalmente a partir del planteamiento de Castoriadis sobre la institución de los significados imaginarios sociales.
\end{abstract}

PALABRAS CLAVE: TEORÍA * CONOCIMIENTO * FILOSOFÍA $*$ CULTURA * IDENTIDAD * CREENCIA $*$ COMPORTAMIENTO SOCIAL

\section{ABSTRACT}

The current essay is an interpretation of the social construction of meanings from the eclosion of the Life -World in contemporary post-modernity. Also, I propose that this eclosion makes possible an epistemological leap that could further the transit from "the kingdom of necessity to the kingdom of liberty" in the dominions of culture and subjectivities. I do this mainly using Castoriadis' vision on the social construction of imaginary meanings.

KEYWORDS: THEORY* KNOWLEDGE $*$ PHILOSOPHY $*$ CULTURE $*$ IDENTITY *BELIEF * SOCIAL BEHAVIOUR

* $\quad$ Centro de Investigación en Identidad y Cultura Latinoamericanas (CICLA) de la Universidad de Costa Rica.

mflorezestrada@racsa.co.cr 
1. EL DESEO DEL PADRE COMO CONSTRUCCIÓN DE SIGNIFICADOS VERDADEROS

Partamos de la pregunta de Castoriadis: "¿Qué es la "unidad" y la "identidad”, es decir, la ecceidad de una sociedad, y qué es lo que mantiene unida a una sociedad?". Y de su propia respuesta a esta pregunta: "Lo que mantiene unida a una sociedad es el mantenimiento conjunto de su mundo de significaciones" (1989: 313).

Nótense dos elementos especialmente significativos: el concepto de "unidad" social y la definición tautológica y práxica de la respuesta, asociada nuevamente, a la idea de unidad o conjunción: "Lo que mantiene unida a una sociedad es el mantenimiento conjunto (...)".

Tampoco debe pasarse por encima la relativización tanto del concepto de unidad como del de identidad, que Castoriadis entrecomilla, consciente de su precariedad.

La unidad social es, pues, relativa: parece existir y la suponemos, en tanto no ocurra la disgregación: estamos juntos porque estamos juntos. Pero, como el propio Castoriadis ha dicho en un momento anterior, ni siquiera cuando se producen graves conflictos sociales o "rupturas" culturales, dejan estas de darse al interior de matrices de significación que nos son comunes:

Lo que es no puede ser caos absolutamente desordenado. (...) Si lo fuera, no se prestaría a ninguna organización, o bien se prestaría a todas: en los dos casos, no sería posible ningún discurso coherente ni ninguna acción. Si se adopta de manera absoluta $y$ radical la tesis empirista-escéptica, lo pulveriza todo, incluso la esperanza que quien la enuncia tiene de que el otro (o él mismo) comprenda lo que dice, oiga los sonidos que profiere, o incluso que exista: si se la considera en sentido relativo, forzosamente ha de dejar espacio a hábitos del sujeto (Castoriadis, 1989: 285-286).

Está pues, la tradición (para Gadamer), o el trasfondo (para Searle), o el habitus (para Bourdieu) o el magma de significaciones sociales imaginarias, para el propio Castoriadis, que son, a la vez, la "amalgama" y el terreno de batalla en el cual la tradición y la imaginación radical disputan la simbolización de ese mundo social.

Pero, ¿qué es simbolizar? Simbolizar es nombrar, mediante la palabra, los hechos o cosas sociales (como diría Durkheim), y en esa medida es instituirlos como significados imaginarios sociales (comunes para todos). El terreno de batalla es el campo de la institución. Es decir, que el lenguaje social $-y$ lo que, por ese mismo carácter común/comunitario, Castoriadis llama la lógica identitario-conjuntista-, instituye el mundo imaginario social y sus límites, al mismo tiempo lo pensable/representable y lo impensable/irrepresentable. Parafraseando su tautología práxica, la sociedad se instituye por medio de instituir un mundo de significaciones: tanto las del representar-decir social (legein) como las del hacer social (teukein).

Nada escapa a la institución social y nadie se escapa de ella aun cuando la repudie o la problematice. Esto, porque incluso en cuanto a la "eclosión de lo identitario" (Castoriadis, 1989: 285) las significaciones imaginarias sociales tienen que ser "participables":

(...) los individuos son formados como individuos sociales, con capacidad para participar en el hacer $y$ en el representar-decir social, que pueden representar, actuar y pensar de manera compatible, coherente, convergente incluso cuando sea conflictual (el conflicto más violento que pueda desgarrar a una sociedad presupone aun una cantidad indefinida de cosas "comunes" o "participables") (Castoriadis, 1989: 323).

Es decir, que socialmente no puede pensarse lo socialmente impensable, o lo que es lo mismo, lo impensable social solo puede pensarse desde lo pensable social (el horizonte que porta la tradición del caminante situado de Gadamer): lo simbólico debe tener sentido (para alguien), y todos somos del-para el magma social de significaciones imaginarias, pues es este la "amalgama" que sostiene y orienta la sociedad en cuestión, es la que, precisamente, da sentido común, lo impone. 
En relación con el lenguaje como estructura básica de la significación y de su institución social, lo anterior implica que hay "unidad" social porque no se puede romper la cadena de significantes. Esto es lo mismo que decir que no puede pensarse lo impensable, o lo que no es participable. Y son, precisamente, la tradición, el trasfondo, el magma de significaciones sociales imaginarias que instituyen los límites de lo imaginario social, los que dan sentido a los significantes. Pregunta Castoriadis: “¿Habría papeles si no hubiera una pieza teatral? ¿Y cómo habría papeles si el conjunto de ellos no formara una obra? ¿Qué obra y quién la ha escrito?"(1989: 232).

Evidentemente, la obra de la lógica identitario-conjuntista, es la obra de Dios-Padre, o como plantea Castoriadis, de la filosofía tautológica o de la verdad en tanto tautología: "En cierto sentido, el verdadero fondo de la gran sofística es el mismo que el verdadero fondo de la filosofía heredada: la exigencia de la tautología; recordemos que, en lógica moderna, verdad se dice tautología" (1989: 299).

En la medida que es el Padre quien habla e instituye, esa clasificación (conjuntiva) no es otra cosa que producción arbitraria (tautológica) de un orden de relaciones identitarias de poder: el orden social se instituye en el nombre del Padre, quien es el ser indeterminado: "es el que es" bíblico1.

Es la forma de violencia más "pura" —el mero poder-, que no es ya la violencia de la fuerza bruta sino de la mucho más sofisticada violencia de lo social institucionalizado (o violencia simbólica, como diría Bourdieu, o violencia epistémica, como diría Spivak):

Por cierto que no se trata de violencia física, sino de violencia del discurso, de la utilización exclusiva y despiadada de la lógica identitaria, que es una exigencia esencial del discurso una vez planteada la cuestión de la determinidad y de la coherencia - es decir, en realidad, desde el

Esta misma lógica es la que, como desarrollo del psicoanálisis, llevó a Lacan a plantear la metáfora del Nombre del Padre, es decir, como interiorización de las estructuras sociales/sexuales, de origen patriarcal ("complejo de Edipo", en Freud), por los sujetos, mediante el lenguaje. primer día del lenguaje- $-y$ que arruina inevitablemente el discurso mismo, pues en este aquella exigencia no puede satisfacerse (Castoriadis, 1989: 299).

Como toda institución social de lo pensable-representable y de sus límites, la lógica identitaria es violencia institucionalizada, pero desde mi punto de vista, su historicidad, esto es, la posibilidad de pensar al Padre, a su lógica identitaria, a su discurso y a sus instituciones, como creaciones sociales imaginarias espaciotemporales - inada menos que pensar al PadreVerdad en su determinidad!-, constituye el primer salto o clivaje epistemológico importante de la historia humana, y es inaugurado -aunque no con plena conciencia de sus implicaciones-por la Modernidad.

Ha sido Foucault (1985) quien ha destacado la importancia del 'salto epistemológico' que significó el abandono del pensamiento antiguo, basado en la similitud y la mimesis relación identitaria, tautológica de la marca, y su sustitución, hacia el siglo XVII, por la representación. Y este 'quiebre' entre las cosas y su marca o nombre (palabra), ocurrió precisamente en el terreno del lenguaje.

A decir de Foucault, el lenguaje se elide en el momento cuando el pensamiento míticosagrado antiguo, basado en la autoría divina que solo puede ser revelada por el exégeta (habilitado para interpretar el verdadero sentido de lo instituido), es reemplazado por el discurso, en tanto posibilidad abierta, del lenguaje, de producir significación. Es el paso, dice Foucault, del comentario a la crítica.

En adelante, las palabras no tienen un valor intrínseco ni coherencia, sino que las adquieren de manera relativa al orden o sintaxis que ocupan en el discurso (en el discurrir de las palabras es cuando se producen los significados). De allí que la nueva epistemología dé nacimiento a la gramática general: el lenguaje como entidad propia, como objeto de estudio (precisamente como la "cosa" social, de Durkheim).

Es sobre esta conciencia racional del carácter convencional de las palabras, de la arbitrariedad y por tanto de la violencia de los signos con que socialmente se nombra el 
mundo, que se funda el pensamiento moderno en Bacon, Descartes, Hobbes, Locke.

Pero no importa tanto, agrega Foucault, cómo se instituyen las palabras, ni lo que representan individualmente, sino cómo su organización o estructura produce los significados: es, por decir así, el discurso el que instituye tanto las palabras como las representaciones, es el dominio, entonces, de los órdenes de discurso.

Castoriadis plantea lo mismo cuando afirma que no hay nombre propio - pues este es, precisamente, la Palabra instituyente del Padre - o "en el principio, fue el Verbo..."-, $y$ por eso afirma que todo lenguaje es siempre abuso del lenguaje.

Y esto ocurre porque el lenguaje no resiste un análisis identitario, en la medida que es siempre tropo o figuración, es siempre transporte de intenciones de significantes que únicamente son en el acto efímero y relativo de la enunciación. Pero, además, la enunciación crea el contexto - pues lo modifica en su acto-y a la vez está condicionada por este, de modo que el significante sólo es no siendo nunca determinable, sólo designa significados volátiles y relativos. De esta forma, la institución, lo instituido, es siempre inacabable $y$ provisional: el lenguaje $-y$ los magmas de los imaginarios histórico socialesson siempre polisémicos inabarcables.

Castoriadis resume así ese inútil combate (como diría Yourcenar), de la filosofía heredada, por hacer valer la epistemología anterior frente a los ataques de los sofistas:

Del mismo modo, Aristóteles, al reconocer la polisemia inabarcable (pollachos legomenon) de los vocablos últimos de la lengua - ser, uno-y al convenir en que las operaciones explícitas de la lógica identitaria están condicionadas, en ambos extremos, por lo que no se deja explicitar en y por esta lógica - "los términos primeros y los últimos, hay nosotros y no logos"-, afirmará que no se puede resistir a los que, en el discurso, solo buscan la violencia (1989: 298).

La cadena de significantes, pues, sólo tiene sentido en la dimensión de lo que Castoriadis llamaba la lógica identitario-conjuntista, donde hay autor, obra y papeles, donde hay identidad o correlación de significante y significado, y cuando se rompe esta cadena - como es en el caso de la psicosis, a decir de Lacan-, lo que hay es locura, incapacidad de simbolizar, pasaje al acto (se ha perdido la dimensión de la participabilidad en lo simbólico que es condición sine qua non de la "unidad" social). Pero a esto volveré más adelante.

Puede afirmarse entonces, con la vasta obra de Foucault, que la Modernidad a la vez nos libera de la Obra del Padre - con la institución del "yo" sujeto de derechos-, pero que, en el mismo acto, $y$ sin pleno conocimiento todavía de las implicaciones de su emancipación del Padre y de la lógica identitaria, vigilará, castigará, encerrará y "curará" a la locura mediante sus propias instituciones imaginarias y sociales.

En términos de tropos o figuras del lenguaje, esto mismo se manifiesta en una revaloración de la metonimia frente a la metáfora. Mientras que la metáfora es una transposición del significado, lo cual pasa por la existencia de una correspondencia entre este $y$ su significante $-y$, por tanto, de una matemática de equivalencias-, en la metonimia se produce un desplazamiento de significados desde un significante hacia otro significante que le es, en parte, próximo:

No existe el "sentido propio"; lo único que existe - pero siempre, e ineliminablemente, ya sea en las metáforas como en las alegorías más sutiles o más disparatadas - es referencia identitaria, punto de una red de referencias identitarias, aprehendido él mismo en el magma de las significaciones y referido al magma de lo que es. ¿Hay una atribución que no sea metonímica? Decir que la hay equivaldría a decir que existen atribuciones o predicaciones absolutas. Pero, ¿qué puede ser una atribución absoluta? En el límite, no puede ser otra cosa que la atribución de la ousia a la ousia, a saber, la tautología absoluta, la forma vacía de la identidad consigo mismo (Castoriadis, 1989: 295).

Se ha roto pues, epistémicamente, con el orden instituido de las cosas, aunque en el 
magma de significaciones imaginarias sociales los significantes identitario-conjuntistas y sus referentes - las significaciones segundas y las significaciones centrales de Castoriadis-, circulen, ahora, como locas.

\section{2. ¿CUÁL ES EL SIGNIFICADO SOCIAL Y CULTURAL DE LA ACTUAL AVERSIÓN TEÓRICA A LA POST-MODERNIDAD?}

En sentido histórico-social, el magma de Castoriadis ya es, pues, post-moderno y, en términos estéticos, kistch: “(...) un haz indefinidamente embrollado de tejidos conjuntivos, hechos de materiales diferentes $y$, no obstante, homogéneos, por doquier tachonado de singularidades virtuales y evanescentes" (Castoriadis, 1989: 289).

La nueva episteme, que ha hecho posible la emancipación del Padre, ya no se estructura en la cadena sintagmática de la identidad y la coherencia, sino en la de la, productiva, difference derrideana (Derrida, 2003). En un proceso de resistencia y maleabilidad indisociables - como propone Castoriadis-, ha cambiado la lógica de la institución de las significaciones imaginarias sociales, pues la institución de la ley se ha vuelto performativa. Es decir, que conserva algo de la tradición, del trasfondo, del magma instituido, a la vez que produce significaciones inesperadas, "fuera de la Ley", como una composición más o menos caótica de fragmentos de ambas lógicas epistémicas, de ambos imaginarios sociales en una nueva "unidad"-siempre precaria, "junta", ambivalente-que presentifica el porvenir.

Como puede deducirse fácilmente, lo anterior es de la mayor importancia particularmente para todo aquello que en las epistemes anteriores - tanto antigua como modernafue dejado fuera de lo simbólico, más allá de los extremos del ser $y$ del lenguaje; de todo aquello omitido como impensable-irrepresentable porque no era más que naturaleza o no logos, en su estado íntegro o monstruoso.

Si en las viejas epistemes hay, a decir de Castoriadis, significaciones que son referentes implícitos, pues ellas dan forma, contenido, significado no explicitados — como son las "significaciones centrales"-, en la post-modernidad actual sólo podría hablarse de la existencia de significaciones "segundas", pero que ahora no son ordinales porque ya no hay "orden" jerárquico. $\mathrm{O}$, en todo caso, de un momento magmático en el cual las significaciones "centrales" de las viejas epistemes fluyen revueltas con las significaciones "segundas" de la nueva episteme, disputándoles el poder simbólico.

En la post-modernidad, significaciones antes "centrales" como "la familia", "la mujer", "la madre" o "la identidad sexual", se vuelven "segundas", pero conviven $-y$ batallan-, por recuperar su centralidad simbólica, con las supuestamente segundas: más aun, por expulsarlas de lo simbólico, devolverlas a los extramuros, al no logos, con el propósito de preservar de manera exclusiva su poder instituyente social.

Sin embargo, es precisa e indeteniblemente desde el "mundo de la vida", de las tematizaciones "ilegales", "fuera de la Ley", de lo marginado y abyectado - es decir, por ejemplo, desde las mujeres (teorías y movimientos feministas), desde los "desviados" o "degenerados" (teorías y movimientos de las sexualidades no heterosexuales, ni binarias)_, y no desde el mundo del pensamiento tematizado hegemónico, desde donde se ha producido la acción de llevar la eclosión de la lógica identitaria hasta sus "últimas" consecuencias, dando paso a una expansión del universo de lo pensable-representable que a su vez presiona los límites de lo simbolizable mucho más allá de lo inicialmente pensable.

Se trata, a mi juicio, de otro salto epistemológico, extremo, de libertad infinitamente elástica, que tensiona nuestro imaginario social a límites casi imparticipables, sin que pueda, ya, llamarse a esto, "locura". Y no puede nombrarse así porque la infinita productividad de las cadenas sintagmáticas relativiza la "sanidad", toda sanidad.

Entonces, también aquí, en la era de la post-modernidad, cabe preguntar: ¿qué es lo que mantiene "unida" a una sociedad?

Si lo que la mantiene unida es el mantenimiento conjunto de su mundo de significaciones, si estamos juntos porque estamos juntos, ¿cómo ocurre actualmente esto y qué es, hoy, estar "juntos"? 
Quizás se entienda mejor si introducimos la idea de que se ha remontado la participabilidad de lo simbólico como condición de lo instituyente, que se le han ampliado extremadamente los márgenes, al punto de que, siendo imparticipable para algunos, tiene, de antemano, su propio estatuto de legitimidad. Y este sería, precisamente, el rasgo más característico de la eclosión epistémica de la post-modernidad: la inclusión de la posibilidad de lo imposible en la cadena significante. Esto, a su vez, implica que se ha expandido el horizonte de la imaginación radical de Castoriadis, que es, precisamente, la que escapa al mundo común social, al sentido común.

Si alegóricamente, en la psicosis hay ausencia de un significante, si la cadena sintagmática se rompe porque no hay posibilidad de simbolización, ¿lo "propio" de la post-modernidad es que puede "decirse"-representarse la ruptura de la cadena significante? En la episteme actual, ¿puede "decirse" el significante (antes) ausente sin el peligro de caer en la locura?

En la post-modernidad, el campo de batalla sigue siendo el campo de la institución. Sigue cumpliéndose que "el imaginario social es histórico y se presentifica y figura en y por la institución" (Castoriadis, 1989:326). Y actualmente también la cadena significante tiene significación, existencia, gracias a la significación instituida por relaciones desiguales de poder. Pero, en esta justa o refriega por hegemonizar lo instituyente, lo que en la antigua episteme era pasaje al acto, locura, hoy ha adquirido carta de ciudadanía epistémica.

Lo que antes había sido erradicado más allá del límite de lo decible-pensable-representable, lo que había sido negado como insimbolizable, hoy se vuelve participable y compite por la institución de los significados imaginarios sociales, y se ha vuelto, de un modo bizarro, "participable" en tanto significante, puesto que ya no hay nombre propio: se han corrido los límites del universo, que son permanentemente expandidos con cada acto de enunciación, en tanto producción de significados sociales.

Este contexto (aun si nos limitamos al contexto estrictamente lingüístico), no puede definirse rigurosamente ni de manera unívoca; en el mejor de los casos, se podría compararlo con una familia de afinidades que cubre una inmensa parte del lenguaje considerado. En términos estrictos, el contexto lingüístico de una frase es la totalidad del lenguaje en el cual es pronunciada, así como su contexto no lingüístico, el universo entero (Castoriadis, 1989: 300-301).

Pero, si en la episteme precedente el contexto tiene una coherente identidad tautológica —es una "familia de afinidades"-, este rasgo siniestro del magma de significados imaginarios sociales presentifica ya su desaparición en el reino de la libertad significante y significativa.

Porque, entonces, podemos decir que, en la episteme post-moderna, es lo reprimido social lo que ha "vuelto": aquello que, a decir de Jiménez (2008a y 2008b), estaba faltando en la foto familiar ${ }^{2}$, pero que siempre estuvo allí como ausencia, como "doble" ambivalente.

Estamos, entonces, en la era de lo reprimido que vuelve, $y$ que Freud (2007) ha descrito como lo siniestro:

(...) lo angustioso, es algo reprimido que retorna. Esta forma de la angustia sería precisamente lo siniestro, siendo entonces indiferente si ya tenía en su origen ese carácter angustioso, o si fue portado por otro tono afectivo. En segundo lugar, si esta es realmente la esencia de lo siniestro, entonces comprenderemos que el lenguaje corriente pase insensible-

2 El autor ha elaborado acerca de cómo lo que él llama el "nacionalismo étnico metafísico" de las élites construyó imaginariamente a la "gran familia costarricense" principalmente a partir de su "blanqueamiento", a su vez asociado a los valores racionales de la Modernidad, lo cual pasa necesariamente por excluir-abyectar de la memoria imaginaria e histórica a una parte de la familia (a la parte, añado, que metafóricamente correspondería, por ejemplo, a "la loca del desván", es decir, a ese integrante de la familia — en la metáfora, principalmente mujeres - que la familia esconde por ser motivo de vergüenza, sea por ser producto de violación, incesto, o porque son, de alguna manera, "anómalos"). 
mente de lo "Heimlich" ("familiar". Nota de MFE), a su contrario, lo "Unheimlich", pues esto último, lo siniestro, no sería realmente nada nuevo, sino más bien algo que siempre fue familiar a la vida psíquica y que sólo se tornó extraño mediante el proceso de su represión. Y este vínculo con la represión nos ilumina ahora la definición de Schelling, según la cual lo siniestro sería algo que, debiendo haber quedado oculto, se ha manifestado (Freud, 2007: 2498) ${ }^{3}$.

En el plano psicoanalítico se trata, precisamente, de la ambivalencia de los significantes (metonimia) a que hizo referencia Castoriadis: "De modo que heimlich es una voz cuya acepción evoluciona hacia la ambivalencia, hasta que termina por coincidir con la de su antítesis, unheimlich. Unheimlich es, de una manera cualquiera, una especie de heimlich" (Freud, 2007: 2488. Las cursivas son del original).

La episteme de la post-modernidad ha hecho participable, pues, un mundo de significaciones sociales que incorpora la dimensión siniestra de la cultura y que estaba reprimida en las epistemes anteriores.

Detallando aún más el contenido de lo siniestro, Freud escribe:

Pero no sólo este contenido ofensivo para la crítica yoica puede ser incorporado al "doble", sino también todas las posibilidades de nuestra existencia que no han hallado realización y que la imaginación no se resigna a abandonar, todas las aspiraciones del yo que no pudieron cumplirse a causa de adversas circunstancias exteriores, así como todas las decisiones volitivas coartadas que han producido la ilusión del libre albedrío (Freud, 2007: 2494).

¿Y qué más "circunstancias exteriores" represoras del yo o coerciones de las decisiones

3 "Se denomina UNHEIMLICH todo lo que debiendo permanecer secreto, oculto... no obstante, se ha manifestado" (Schelling, 2, 2, 649), citado por Freud, 2007: 2487. volitivas productoras de la ilusión del libre albedrío, que las reglas culturales, comenzando por las que permiten y reprimen, en el mismo acto, la simbolización del "universo" y de sus límites?

Si nos limitáramos a analizar las consecuencias de este cambio radical para el discurso psicoanalítico, quedaría claro que ha llegado el fin del "complejo de Edipo", en tanto metaforización a la vez del "complejo de castración" o tabú del incesto y del asesinato del Padre, sobre el que, en la interpretación freudiana, se funda la cultura. Igualmente, queda relativizado todo diagnóstico e interpretación de la psicosis como ausencia o ruptura en la cadena de significantes $y$, desde mi punto de vista, se abre todo un "mundo" de posibles nuevas interpretaciones ${ }^{4}$.

Pero de un modo más general, remite a la pregunta: ¿qué es lo real y qué lo imaginario en el mundo-magma de significaciones sociales cuando lo reprimido, ahora participable, vuelve con su consustancial ambivalencia? ¿No nos encontramos, más bien, en un nuevo momento de redefinición de la producción tanto de lo imaginario como de lo real?

Evidentemente, la respuesta a esta pregunta puede generar, también, emociones ambivalentes. Por una parte, la vieja aversión hacia lo siniestro hace temer acerca del futuro de la "unidad" social y propicia las profecías catastrofistas de las cuales se culpa a la postmodernidad en tanto expresión cultural del capitalismo avanzado. Por el otro, la apertura de la conciencia hacia lo "nuevo" — que, por otra parte, no lo es tanto, pues precisamente porque es a la vez familiar/heimlich, nos resulta extraño/unheimlich, o en inglés, uncanny-, no deja de presentificarnos un mundo de mayor felicidad, en tanto menor represión, exclusión,

Durante las Jornadas de Investigación del año 2008, en el Instituto de Investigaciones Sociales de la UCR, sugerí la posibilidad de considerar, en algunas circunstancias, el pasaje al acto incluso en los casos de asesinato como un síntoma saludable, como un salvarse a una misma frente a la locura de la cultura, $y$ no a la inversa. En este caso lo plantee en relación con una mujer acusada de infanticidio - estudiado por la Dra. Laura Chacón-, quien luego de cumplir su pena en la cárcel rehízo su vida junto con su pareja, esta vez una mujer. 
discriminación y posibilidad de reintegración del "yo", individual y cultural.

Curiosamente, por sus lazos más arcaicos con los conceptos estamentales de la episteme antigua, mucho del pensamiento marxista contemporáneo $^{5}$, sea en la filosofía como en los estudios culturales (pienso en Fredric Jameson: El posmodernismo o la lógica cultural del capitalismo avanzado, 2008), comparte mucho más que el pensamiento de origen liberal moderno, los temores a una disgregación social o "barbarie", como si tal disgregación fuese posible (recuérdese que ni estamos realmente unidos, ni nos queda otro remedio que estar "juntos").

En esa obra, Jameson, por ejemplo, analiza con nostalgia y preocupación la estética de la post-modernidad en el capitalismo estadounidense y asocia la ruptura de la cadena de significantes con un proceso de esquizofrenia - lo metonímico como esquizofrenia y no como proceso creativo, que es lo que yo propongo-, con lo cual pierde de vista a la post-modernidad como un momento precisamente ambivalente y dinámico, que puede resultar amenazante para quienes se han sentido incluidos en la episteme moderna - desde todos los hombres heterosexuales no "dementes" hasta el "proletariado"-, pero pierde de vista el poder revolucionario que la nueva episteme implica para quienes siempre estuvieron, como dije, en los extramuros de la Modernidad.

$Y$ esto ocurre precisamente cuando, en términos epistemológicos, la post-modernidad más bien presentifica un acercamiento del "reino de la libertad" al que aspiró el joven Marx de los Manuscritos (1844). Paradójicamente a la percepción marxista ortodoxa $-y$, en general, al sentido común de las ciencias sociales-, estaríamos viviendo, entonces, un momento histórico en el cual la superestructura - la cultura - camina, se transforma, más rápido o al menos tan rápido como la infraestructura, $y$ esto es percibido de manera distinta, como

Como he planteado en otros trabajos, me refiero a conceptos estamentales típicos del mundo antiguo como los de "clase", "conciencia de clase" o "proletariado", que además tienen sentido en una visión teleológica del mundo. amenaza o como promesa, según se sea o no sujeto de una particular violencia epistémica. (¡Así vemos nada menos que al revolucionario ortodoxo por excelencia temiendo a las transformaciones grandiosas del capitalismo cuyo potencial liberador el propio Marx nunca perdió de vista $y$, por el contrario, a partir de cuyas potencialidades o presentificaciones elaboró su programa!).

Lo cierto es que los análisis que insisten en la ortodoxia para interpretar el "capitalismo tardío" o el "post-capitalismo" bien necesitarían una revisión a fondo. Quizás ciertos conceptos $y$ ciertos métodos de interpretación que todavía nadan en el magma de significaciones sociales de la post-modernidad, son simplemente inservibles, ya, no sólo para interpretar, sino para transformar, el mundo.

\section{BIBLIOGRAFÍA}

Bourdieu, Pierre. Masculine domination. Stanford, California. Polity Press: Stanford University Press, 2001.

Castoriadis, Cornelius. "La institución imaginaria de la sociedad". Volumen segundo. El imaginario social y la institución. Barcelona. Tusquets, 1989: 283-334.

Derrida, Jacques. Márgenes de la filosofía. Madrid: Cátedra. Teorema, 2003.

Foucault, Michel. Las palabras y las cosas. $16^{a}$ Edición. Siglo Veintiuno Editores, 1985.

Freud, Sigmund. "Lo siniestro". Obras completas. Tomo 3 (1916-1938) [1945]. Madrid. España: Biblioteca Nueva, 2007.

Gadamer, Hans-Georg. Verdad y método. $7^{\mathrm{a}}$. Edición. Salamanca: Sígueme, 1997.

Husserl, Edmund. "La filosofía en la crisis de la humanidad europea”. Husserl, E. 
Invitación a la fenomenología. Barcelona. Paidós, 1992: 75-128.

Jameson, Fredric. El posmodernismo o la lógica cultural del capitalismo avanzado. Barcelona: Paidós Studio, 2008.

Jiménez, Alexander. Ese otro país al cual seguimos llamando Costa Rica. (El momento de los lazos sociales). Exposición ante el Congreso de Salud Mental. San José. Octubre. 2008a. Copia impresa facilitada por el autor.

Jiménez, Alexander. El imposible país de los filósofos. San José: Editorial de la Universidad de Costa Rica, $2008 \mathrm{~b}$.

Marx, Karl. Manuscritos económicos y filosóficos. En:<www.marxists.org/ espanol/m-e/1840s/manuscritos/man2. htm>. [Consultado el 15/07/2009].

Searle, John R. La construcción de la realidad social. Barcelona. Buenos Aires. México. Paidós, 1997: 139-158.

Spivak, Gayatri Ch. ¿Puede hablar un subalterno? Buenos Aires, Argentina: Centro de documentación sobre la Mujer. En: <www.libreriademujeres.com.ar $>$ 
